\title{
Collapsing process simulations of timber structures under dynamic loading II: simplification and quantification of the calculating method
}

\begin{abstract}
In a previous paper we presented the efficacy of the extended distinct element method (EDEM) on the collapsing process simulation of timber-frame structures. The results obtained were qualitative, and the need for quantitative simulation was pointed out. In this report, we improved our simulating programs with the aim of establishing a quantitative simulation by EDEM. For this purpose, the parameters of the frame members (beam or column) were determined by comparing the simulated results of fourpoint bending tests with experimental results. Other parameters of plywood and joint members were determined by simulations of two rail shear and slip tests of joint parts, respectively. Using these parameters we made simulations of the collapsing process of an actual-size plywoodsheathed wall and at the same time carried out the experiments under similar conditions. Simulated load-displacement curves of the plywood-sheathed wall were similar to those obtained in the experiments, and the sequence of failure at nailed joints of the simulation model gave good agreement with that of the experimental collapsing processes. Using these processes, we believe that we established a basic quantitative simulation method to test the collapsing process of timber-frame structures.
\end{abstract}

Key words Shear wall • Distinct element method . Computer simulation - Timber-frame structure

Takafumi Nakagawa ( $\square) \cdot$ Masamitsu Ohta

Graduate School of Agricultural and Life Sciences, The University

of Tokyo, 1-1-1 Yayoi, Bunkyo-ku, Tokyo 113-8657, Japan

Tel. +81-3-5841-5253; Fax +81-3-5684-0299

e-mail: nakagawa@a.fp.a.u-tokyo.ac.jp

Part of this paper was presented at the 52nd Annual Meeting of the Japan Wood Research Society, Gifu, April 2002; and at the 7th World Conference of Timber Engineering, Malaysia, August 2002

\section{Introduction}

To improve the performance of wooden structures, it is important to know the fracture behavior of the structures under various loading conditions. For this purpose, the establishment of a simulating method to explore the fracturing process is desirable.

In our previous report ${ }^{1}$ we developed a simulating method based on the extended distinct element method (EDEM). There, we made three-dimensional simulations for two-story frame construction models and obtained good simulated results that correspond to the experimental one qualitatively. To obtain a quantitative output from the simulation, however, it was pointed out that the relations between the parameters of spring elements and actual mechanical properties need clarification.

In this study we examined the parameters of EDEM spring elements needed to obtain quantitative results. We also tried to reduce the number of elements of structural members and joint parts in the simulation model for the convenience of large-scale simulation models that correspond to actual wooden houses.

\section{Theory}

As the principle of EDEM was precisely explained in our previous report, ${ }^{1}$ we explain only the modified points here. For analysis of the whole structure it is desirable to reduce the number of elements used in the calculation. In our previous report, frame members consisted of four particle elements in the cross section connected by eight spring elements, including diagonal springs. Thus, it was difficult to develop our models based on the large-scale model, as it would require large memories and long calculating times.

Here, we tried to simplify the configuration of the elements by reducing the number of elements. For this purpose, we used only one particle element in the cross section for the frame members (Fig. 1a); and to resist the bending 

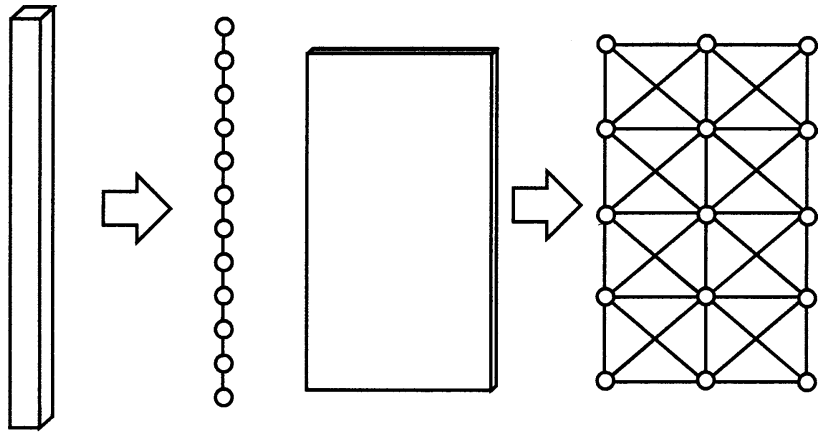

(a) Frame member

(b) Panel member

Fig. 1. Modeling of structural components by particle elements and spring elements

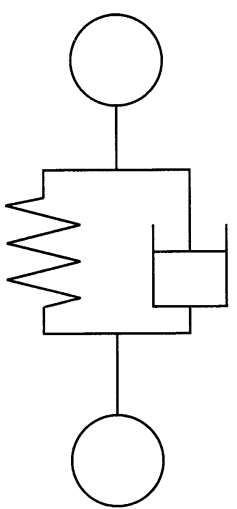

Normal spring

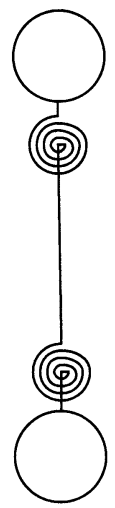

Rotational spring
Fig. 2. Spring elements

moment, we employed a rotational spring instead of shear springs and a diagonal configuration of springs. By this simplification of the spring configuration, we lost the possibility of treating the material orthotopically; but in frame structures the anisotropy of the frame members is generally not so important.

The EDEM elements in this report are depicted in Fig. 2. The normal spring connects particle elements in the normal direction, and the rotational spring works against the relative rotation. Figure 3 shows the forces generated by the rotational spring following movement of particle element $\mathrm{B}$ from the original point $i$ to $i^{\prime}$. The EDEM calculation was made under the following theoretical concepts.

The motion of particle element $i$ having the mass $m$ is expressed as follows

$\boldsymbol{F}_{i}=\boldsymbol{F}_{n i}+\boldsymbol{F}_{s i}$

$m \frac{d^{2} \boldsymbol{u}_{i}}{d t^{2}}+C \frac{d \boldsymbol{u}_{i}}{d t}+\boldsymbol{F}_{i}=0$

where $F_{n i}$ represents the forces from normal springs; $F_{s i}$ is the sum of all forces generated by the relative rotation against adjacent particle elements; $F_{i}$ is the sum of all forces acting on the particle elements; $C$ is the coefficient of viscous damping; and $u_{i}$ is the displacement vector.

The accelerations of the element in $\mathrm{x}$ - and $\mathrm{y}$-directions are then obtained by the following relations

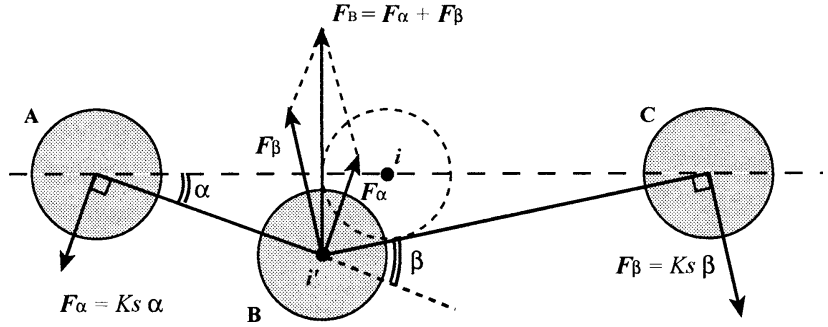

Fig. 3. Forces that act on the rotational spring. A, B, C, particle elements; $K_{\mathrm{s}}$, a constant; $\alpha, \beta$, relative rotational angles; $F \alpha, F \beta$, forces acting on the particle element, generated by the rotational spring

$\frac{d^{2} x_{i t}}{d t^{2}}=\frac{F_{x i t}}{m}, \quad \frac{d^{2} y_{i t}}{d t^{2}}=\frac{F_{y i t}}{m}$

where $F_{x i t}$ and $F_{y i t}$ are the components of the forces acting on element $i$ at time $t$. Velocities and displacement increments of element $i$ during time increment $\Delta t$ are obtained by the numerical integration of these equations.

Following this calculation, we can obtain the coordinates of all particle elements consecutively; thus, we can follow every step of the fracturing process in a computer. The panel members were made up as a lattice sheet of particles that connected each other in the horizontal, vertical, and diagonal directions by spring elements (Fig. 1b).

\section{Determining method of the spring parameters}

In our previous report, the EDEM parameters were determined by the characteristic data of frame members (e.g., modulus of elasticity, cross-sectional area, density) deductively. However, simulated mechanical values by this method were different from the actual ones, and the results obtained had rather poor value from a practical viewpoint. Therefore, we tried to adjust the induced stress and deformation of the EDEM model to the corresponding experimental results at the frame or panel member levels, relating them using the following methods.

\section{Spring parameters of frame members}

We defined spring parameters of frame members by carrying out simulations of four-point bending tests, shown in Fig. 4. As EDEM is a dynamic analysis, it requires many cycles of calculation to obtain a convergent solution by the simulation model, as shown in Fig. 5a. The loaddisplacement curves of each spring parameter can be obtained by changing the loading weight (Fig. 5b). From these curves we obtained a relational equation between the bending modulus of elasticity (MOE) and the parameter of rotational spring $K_{\mathrm{s}}$ as follows

$$
K_{\mathrm{s}}(\mathrm{N} / \mathrm{m})=k_{\mathrm{b}} \times \operatorname{MOE}(\mathrm{GPa})
$$

where $k_{\mathrm{b}}$ is a constant. For the frame member of $10 \times$ $10 \mathrm{~cm}$ cross-sectional area, $k_{\mathrm{b}}$ is $2.03 \times 10^{4}$. The parameters 


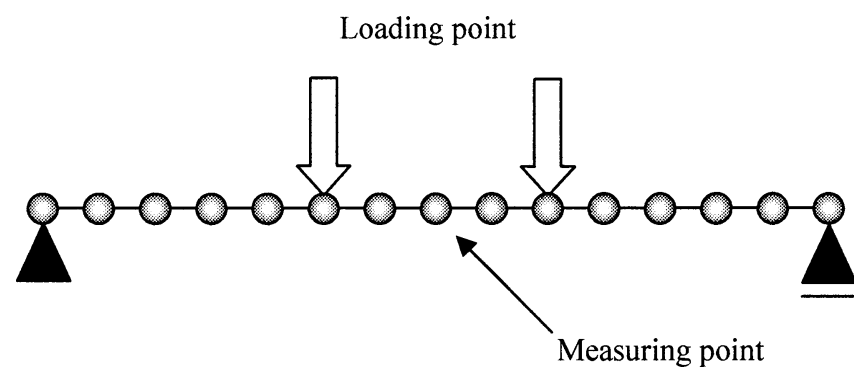

Fig. 4. Simulation of the four-point bending test

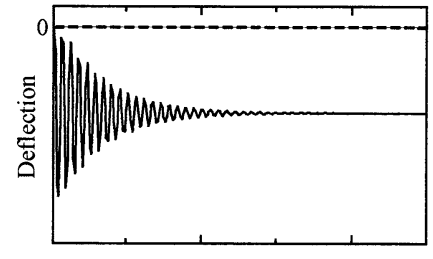

Number of caluculating cycles

(a)

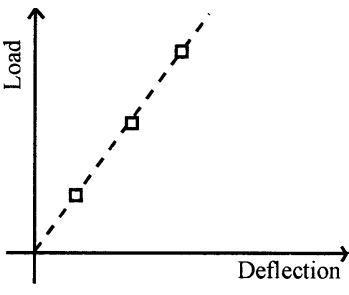

(b)
Fig. 5. Method to determine bending rigidity. a Transition of deflection by extended distinct element method (EDEM) calculation. b simulated load-deflection relation

of a normal spring is defined as: MOE $\times$ cross-sectional area.

Spring parameters of panel members

We defined the parameters for spring elements of the panel members by simulations of the two-rail shear test, as shown in Fig. 6. The same procedure as in the preceding section was carried out to obtain load-displacement curves. As a result, the following relational equation between the shear modulus $G$ and the parameter for a normal spring $K_{\mathrm{n}}$ was obtained

$$
K_{\mathrm{n}}(\mathrm{N} / \mathrm{m})=k_{\mathrm{g}} \times G(\mathrm{GPa})
$$

where $k_{\mathrm{g}}$ is a constant. For plywood of $8.9 \mathrm{~mm}$ thickness, $k_{\mathrm{g}}$ is $5.2 \times 10^{4}$.

\section{Springs parameters of nailed joints}

Spring parameters of the nailed joint were determined by the results of single shearing tests. We approximated loaddisplacement curves of the shear tests under monotonic loading by trilinear curves and adapted them to the nonlinear spring of nailed joints in our simulation models (Fig. 7). Representative data for the CN50 nail corresponding to this curve are shown in Table 1.

Spring parameters of joints between frame members

Spring constants of the joints between frame members were determined by approximating load-displacement curves of

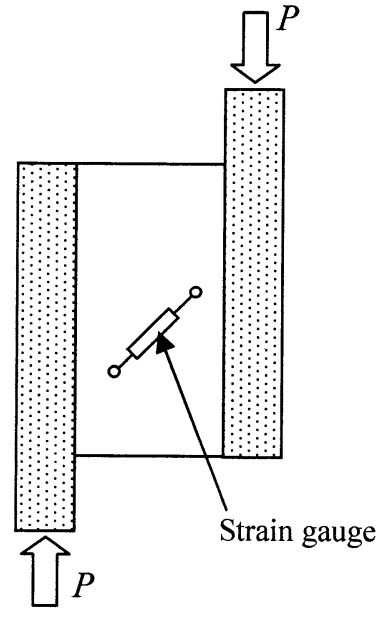

(a)

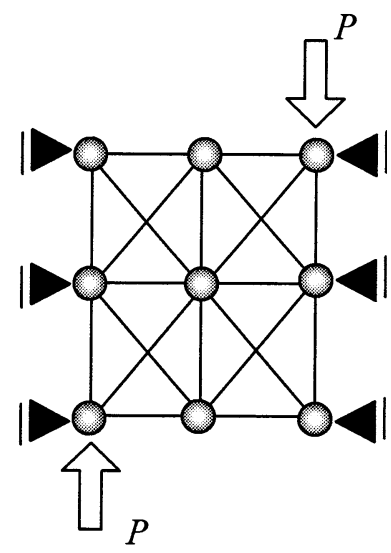

(b)
Fig. 6. Simulation of two-rail shear test. a Schema of two-rail shear experiment. b EDEM model of two-rail shear test

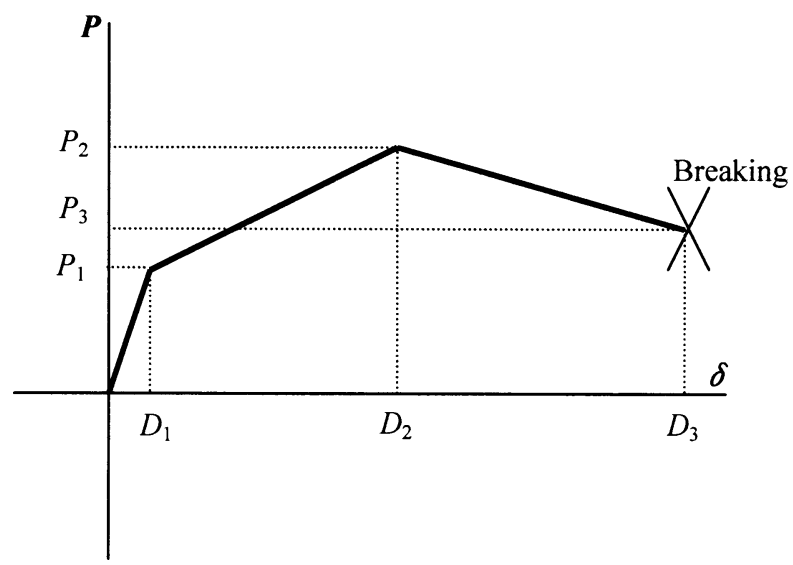

Fig. 7. Nonlinear relation between load and displacement for the nailed joint springs. $P_{1}, P_{2}, P_{3}, D_{1}, D_{2}$, and $D_{3}$ are the specific values on the envelope curve of the tension side (see Table 1)

Table 1. Representative data in the load-displacement curves of nailed joints in the simulation with type $\mathrm{CN} 50$

\begin{tabular}{lc}
\hline Parameter & Result \\
\hline Load $(\mathrm{kN})$ & \\
$\mathrm{P}_{1}$ & 0.75 \\
$\mathrm{P}_{2}$ & 1.75 \\
$\mathrm{P}_{3}$ & 1.25 \\
Displacement $(\mathrm{mm})$ & \\
$\mathrm{D}_{1}$ & 0.6 \\
$\mathrm{D}_{2}$ & 15.0 \\
$\mathrm{D}_{3}$ & 40.0 \\
\hline
\end{tabular}

experimental results under monotonic loading reported in the literature, ${ }^{2,3}$ where the characteristic values for the mechanical properties of the joint part between sill/beam and column with a hold-down fastener (type S-HD20), a corner fastener (type CPT), and a clamp iron (type C120) were reported. Considering embedment, the parameters for the 
Table 2. Representative data in the load-displacement curves of joints between frame members in the simulation

\begin{tabular}{|c|c|c|c|c|c|c|c|c|c|c|}
\hline \multirow[t]{2}{*}{ Fastener type } & \multicolumn{5}{|c|}{ Load $(\mathrm{kN})$} & \multicolumn{5}{|c|}{ Displacement $(\mathrm{mm})$} \\
\hline & $P_{1}$ & $P_{2}$ & $P_{3}$ & $P_{4}$ & $P_{5}$ & $D_{1}$ & $D_{2}$ & $D_{3}$ & $D_{4}$ & $D_{5}$ \\
\hline S-HD20 & 21.5 & 41.4 & 40.0 & 12.0 & 25.0 & 5.19 & 25.0 & 40.0 & 2.00 & 16.0 \\
\hline CPT & 8.16 & 14.2 & 10.00 & 12.0 & 25.0 & 0.690 & 10.0 & 30.0 & 2.00 & 16.0 \\
\hline C120 & 3.20 & 8.50 & 6.00 & 12.0 & 25.0 & 0.500 & 13.0 & 18.0 & 2.00 & 16.0 \\
\hline
\end{tabular}

S-HD20, hold-down fastener; CPT, corner fastener; C120, iron clamp

Data are from Ohno et al., ${ }^{2}$ Nagaoka et al., ${ }^{3}$ and Inayama ${ }^{4}$

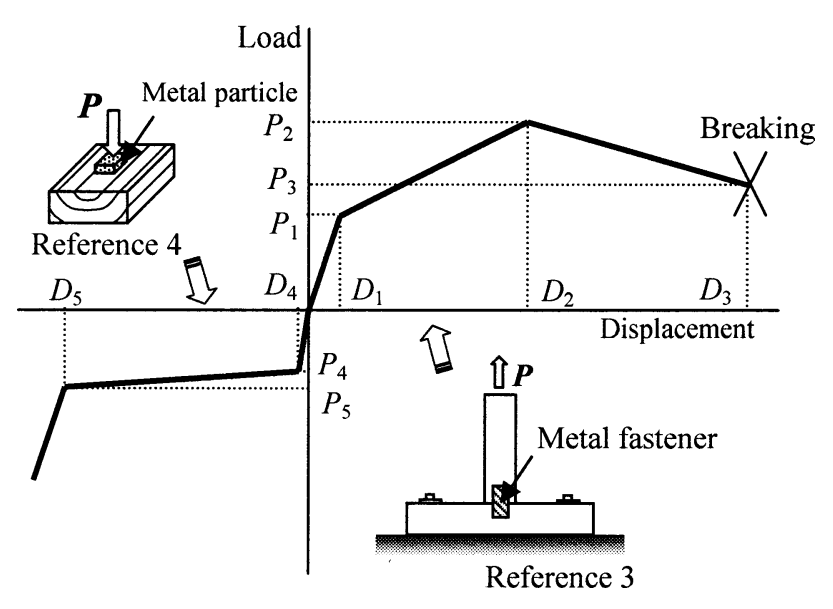

Fig. 8. Nonlinear relation between load and displacement for the joint springs at the joints between frame members. $P_{1}, P_{2}, P_{3}, D_{1}, D_{2}, D_{3}$ (see Fig. 7). $P_{4}, P_{5}, D_{4}, D_{5}$, specific values on the envelope curve of compression side (see Table 2)

compression side were determined using the experimental results under monotonic loading reported in the literature. ${ }^{4}$ Combining two curves obtained from monotonic loading tests reported in the literature, the load-displacement curve of this joint spring is shown in Fig. 8. Representative data corresponding to the curve of Fig. 8 are shown in Table 2.

The value of the damping coefficient $C$ in Eq. 2 was obtained using the following equation ${ }^{5}$

$C=2 h \sqrt{m k}$

where $h$ is the damping factor; and $k$ is a spring constant.

Fracture condition

In the EDEM calculation, the fracture condition is generally defined by the concept of the maximum deformation theory as follows

$D_{i j}>L_{i j}$

where $D_{i j}$ is the distance between the particle elements $i$ and $j$; and $L_{i j}$ is maximum displacement of nailed joints or frame joints in experiments. $L_{i j}$ corresponds to the displacement $D_{3}$ in Figs. 7 and 8. If these fracture conditions are satisfied at any step of the EDEM calculation, spring elements connecting the relating two elements to each other are eliminated.
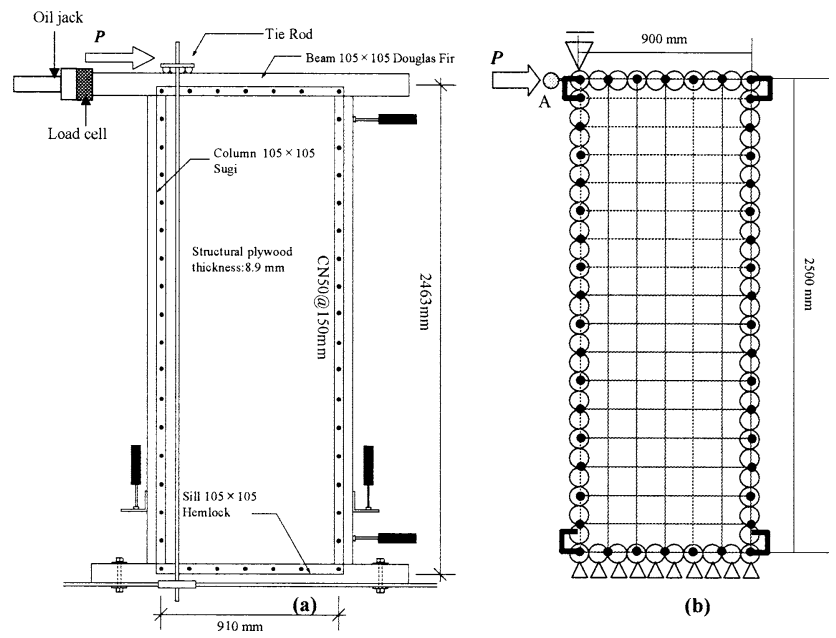

(b)

Fig. 9. Specimen and simulation model of the shear test. a schema for the experimental specimen. b EDEM model (only main elements are shown). Heavy black dots, nailed position; A loading point; heavy $U$ lines, position of the C120 iron clamp

\section{Evaluation of the EDEM simulation by the sheathed shear wall test}

Shear experiments of a simple wood-framed shear wall with plywood sheathing were carried out to verify the validity of our method to deterdmine EDEM parameters. We conducted the EDEM simulation under similar conditions.

Specimens and experimental method

A wood-framed shear wall shown in Fig. 9a was the target specimen. The size and species used for the frame members are shown in Fig. 9a. Specimens were sheathed by a softwood plywood of $8.9 \mathrm{~mm}$ thickness on one side. The nails used were CN50 type (JIS A 5508), 50.8 mm length and $2.87 \mathrm{~mm}$ diameter, and the distance for each nail was $150 \mathrm{~mm}$. Columns were connected to sills and beams by iron clamps (C120). A tie-rod system restrained the upstroke of the specimen. Loading was done at a rate of $5 \mathrm{~mm} / \mathrm{s}$ by an oil jack under monotonic loading until the load decreased to $80 \%$ of the maximum value. Four specimens were tested. 
Simulation model

The simulation model used in our calculations is shown in Fig. 9b. This model assumed a real nailed shear wall. The model was $900 \times 2500 \mathrm{~mm}$. The modulus of elasticity of frame members was set at $7.5 \mathrm{GPa}$, and the shear modulus of the panel member was set at $0.5 \mathrm{GPa}$. To simulate tie-rod system behavior, the upstroke movement of the top left side element was limited by a roller. The reaction force of the model panel was estimated to be the force induced in the normal spring between the top left element and the loading (point A in Fig. 9b), which was added for the convenience of calculation. Nailed points by $\mathrm{CN} 50$ nails were modeled by nonlinear joint springs, and the positions are indicated in Fig. 9b. As the loading is monotonic in our experiment, the same load-displacement relation shown in Fig. 7 was used for all nailed joints. The column and beam/sill were connected by a joint spring simulating a C120 iron clamp. The time increment for a single calculating cycle was $1.0 \times$ $10^{-5} \mathrm{~s}$. Loading was done under monotonic loading until displacement reached $200 \mathrm{~mm}$. The calculated cycles numbered two million.

\section{Results and discussion}

\section{Load-displacement curves}

Figure 10 shows load-displacement curves of the shearing tests of shear walls and that of our simulation. It is apparent that the shapes of these curves are similar. Table 3 shows characteristic values for four experiments and the simulation. These values were calculated from the load-actual shear deformation angle relation based on Japanese structural design guidelines. ${ }^{6}$ The averages of experimental values showed good agreement with the simulated result. Here, the structural characterization factor $(D s)$ indicates the reducing ability of the shearing force by the elastoplastic deformation of the structure. It is defined by the following equations

$$
D s=\frac{1}{\sqrt{2 \mu-1}}, \quad \mu=\frac{\delta_{u}}{\delta_{y}} \frac{P_{y}}{P_{u}}
$$

where $\delta_{u}$ and $P_{y}$ are yield displacement and yield load, respectively; $\delta_{u}$ is the ultimate displacement, which corresponds to the point that the load decreased to $80 \%$ of the maximum load; and $P_{u}$ is the ultimate load. $P_{u}$ is obtained by solving the following equation.

$$
P_{u}\left(\delta_{u}-\frac{1}{2} \frac{P_{u}}{P_{y}} \delta_{y}\right)=\int_{0}^{\delta_{u}} P(x) d x
$$

Symbols used here are depicted in Fig. 11.

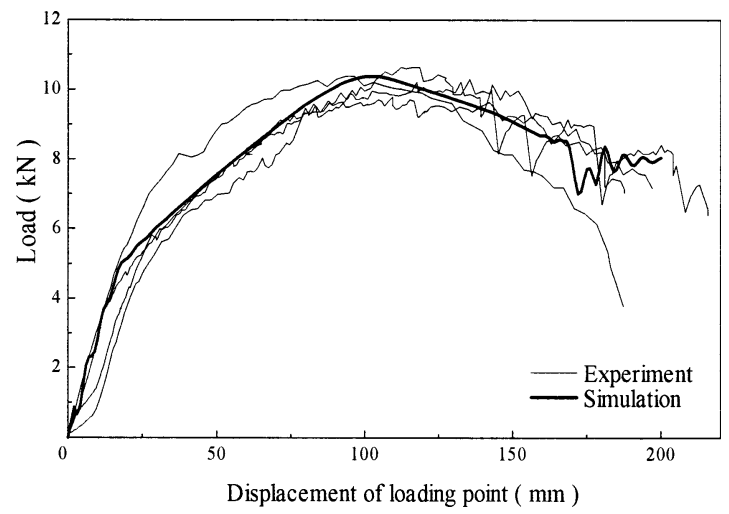

Fig. 10. Comparison of experimental and simulated results of the shearing tests of shear walls

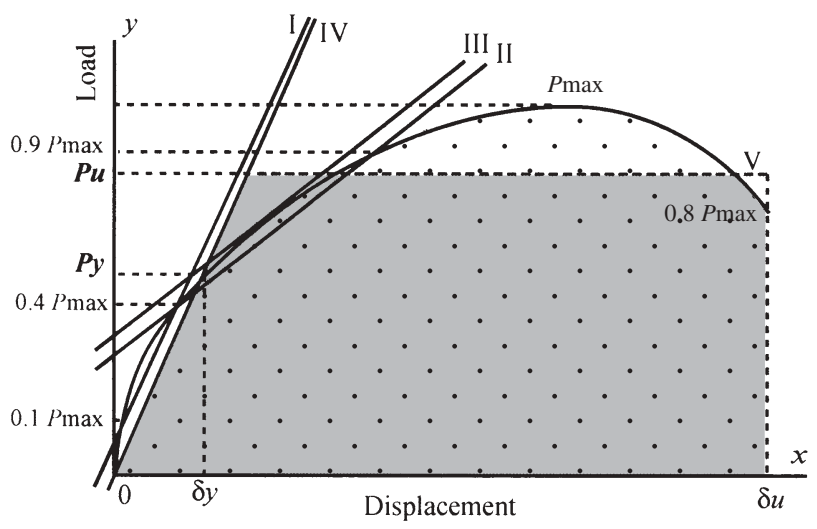

Fig. 11. Definitions for calculating structural characterization factors. $I$, connecting line between the $0.1 P_{\max }$ and $0.4 P_{\max }$ on the loaddisplacement $(1-d)$ curve; $I I$, connecting line between the $0.4 P_{\max }$ and $0.9 P_{\max }$ on the $1-\mathrm{d}$ curve; $I I I$, tangential line to $1-d$ curve parallel to $I I ; P_{y}$, yielding load defined as crossing point of lines $I$ and $I I I ; \delta_{y}$, yielding deformation corresponding to $P_{y}$ on the $1-d$ curve; $I V$, line connecting origin and point $\left(\delta_{y}, P_{y}\right) ; \delta_{u}$, ultimate displacement corresponding to the $0.8 P_{\max }$ on the $1-d$ curve; $V$, line parallel to $x$-axis drawn so the area surrounded by this line, line $I V, x$-axis, and line $y=$ $\delta u$ is equal to the area surrounded by the $1-d$ curve (see Eq. 8); Pu, ultimate load corresponding to line $V$

Table 3. Characteristic values for the specimens of shearing tests of shear walls

\begin{tabular}{llllllll}
\hline Parameter & \multicolumn{2}{l}{ Experiment } & & & & \multirow{2}{*}{$\begin{array}{l}\text { Simulated } \\
\text { values }\end{array}$} \\
\cline { 2 - 6 } & 1 & 2 & 3 & 4 & Ave. & SD & \\
\hline$P_{\max }(\mathrm{kN})$ & 10.2 & 10.6 & 10.4 & 9.79 & 10.3 & 0.355 & 10.5 \\
$P_{\mathrm{y}}(\mathrm{kN})$ & 2.23 & 3.39 & 3.00 & 1.95 & 2.64 & 0.667 & 3.32 \\
$P_{\mathrm{u}}(\mathrm{kN})$ & 8.67 & 8.78 & 9.22 & 8.95 & 8.90 & 0.242 & 8.92 \\
$P_{1 / 150}(\mathrm{kN})$ & 6.00 & 6.11 & 7.19 & 5.58 & 6.22 & 0.684 & 5.64 \\
$D_{s}$ & 0.234 & 0.254 & 0.239 & 0.244 & 0.243 & 0.009 & 0.240 \\
\hline
\end{tabular}

$P_{\max }$ maximum load; $P_{\mathrm{y}}$ yield load; $P_{\mathrm{u}}$ ultimate load of perfect elastoplastic model; $P_{1 / 150}$ load at shear deformation of $1 / 150 \mathrm{rad} ; D_{s}$, structural characterization factor; $1-4$, specimen codes 
Fig. 12. Collapsing process of EDEM simulation model. Arrows indicate breaking points. Numbers are the sequence of the failure
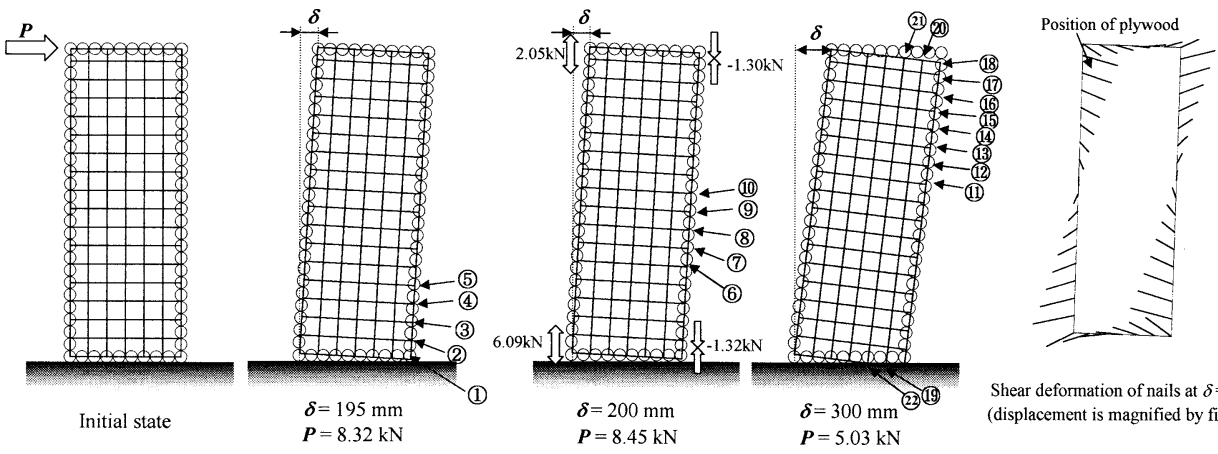

Shear deformation of nails at $\delta=100 \mathrm{~mm}$.
(displacement is magnified by fifty times.) $\delta=300 \mathrm{~mm}$
$P=5.03 \mathrm{kN}$

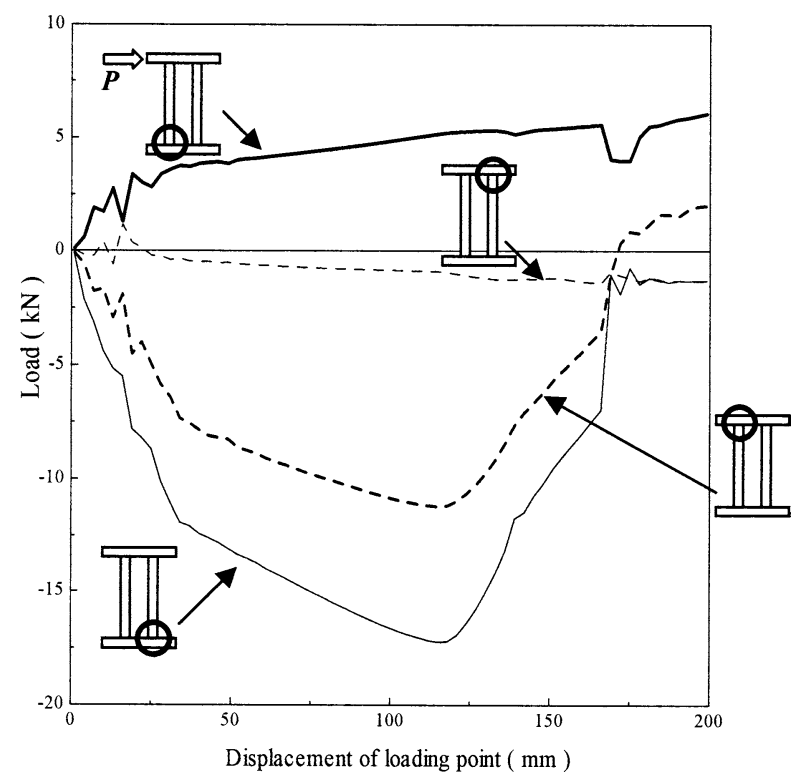

Fig. 13. Load-displacement curves of joint springs corresponding to the C120 iron clamp between the column and the beam/sill. The $x$-axis is equal to the displacement shown in Fig. 10. The positive direction of the $y$-axis indicates the tensile load

The $D s$ value is postulated to be used under cyclic loading conditions, but we adapted it to our experimental results and simulated one under monotonic loading. The $D s$ value obtained from experimental results agrees with the simulated value. Thus, it was found that the ductility of shear walls was also simulated well by this method.

\section{Sequence of failure}

Figure 12 shows the sequence of failure at nailed joints and shear deformation of nails. At first, failure occurred in the nailed joint at the lower right-hand corner of the simulation models, after which the failures spread to adjacent nailed joints. After all the nailed joints of the right side column were broken, failures developed at the sill and beam. Generally, it is difficult to formulate the failure of nailed joints in the actual experiments because failure patterns of nailed joint are various (e.g., punching out, pull out, cutoff of nail). Despite that, the fracture sequence of the model simulation expresses breaking phenomena qualitatively, which corresponds well with the observed results of the actual experiments. Figure 13 shows the load-displacement relations of joint springs between column and beam/sill. The nonlinear characteristic is observed in Fig. 13.

\section{Conclusions}

Simulated load-displacement curves of the plywoodsheathed wall were similar to those obtained in the experiments, and the sequence of failure at nailed joints of the simulation model agreed well with experimental collapsing processes qualitatively. Consequently, it turned out that our newly developed EDEM modeling method and the method to determine EDEM parameters are adequate for analysis of timber-frame structures. This method thus, proved to be useful for quantitative analysis of the fracture process of timber-frame structures.

Acknowledgments This work was supported in part by a Grant-in-Aid for Scientific Research from the Ministry of Education, Science, Sports, and Culture, Japan (grant 09460072), and by a Grant-in-Aid for Scientific Research from the same Ministry (Research Fellowships of the Japan Society for the Promotion of Science for Young Scientists).

\section{References}

1. Nakagawa T, Ohta M (2003) Collapsing process simulations of timber structures under dynamic loading I: simulations of two-story frame models. J Wood Sci 49:392-397

2. Ohno Y, Okabe M, Sakuma H, Shimoyashiki T (2001) Comparison of tie-rod system with hold-down system under horizontal loading test for shear wall (in Japanese). In: Summaries of technical papers of the annual meeting of the Architectural Institute of Japan, C-1, structure III, Tokyo, pp 309-310

3. Nagaoka K, Tsuchimoto T, Ohta M (1999) Fracturing process analysis of joint connections of Japanese frame constructions under different deformation rate. In: Proceedings of the Pacific timber engineering conference, vol 3, Rotorua, pp 303-308

4. Inayama M (1991) The theory and application for crashing in wood (in Japanese). Doctoral dissertation, University of Tokyo

5. Meguro K, Hakuno M (1988) Fracture analyses of concrete structures by granular assembly simulation (in Japanese). Bull Earthquake Res Inst 63:409-468

6. Japan $2 \times 4$ Builders Association (1998) Structural design guidelines for wood frame construction (in Japanese). Japan $2 \times 4$ Builders Association 